\title{
Hypernuclear spectroscopy with extended shell-model configurations
}

\author{
Atsushi Umeya, ${ }^{a, *}$ Toshio Motoba $^{b, c}$ and Kazunori Itonaga ${ }^{d, e}$ \\ ${ }^{a}$ Liberal Arts and Sciences, Nippon Institute of Technology, \\ 345-8501 Miyashiro, Saitama, Japan \\ ${ }^{b}$ Research Center for Nuclear Physics, Osaka University, \\ 567-0047 Ibaraki, Osaka, Japan \\ ${ }^{c}$ Laboratory of Physics, Osaka Electro-Communication University, \\ 572-8530 Neyagawa, Japan \\ ${ }^{d}$ Faculty of Medicine, Miyazaki University, \\ 889-1692 Miyazaki, Japan \\ ${ }^{e}$ Faculty of Education, Gifu University, \\ 501-1193 Gifu, Japan \\ E-mail: aumeya@nit.ac.jp
}

The $\left(e, e^{\prime} K^{+}\right)$experiments done at the Jefferson Laboratory provide us high-resolution structure information on various excited states of hypernuclei. Among others, the $\Lambda$ hyperon plays a unique role of inducing intershell mixing of the positive-parity and negative-parity nuclear core-excited states. In order to elucidate such new phenomena realized in hypernuclei, we have proposed an extended shell model which treats the different-parity nuclear excited states coupled with a $\Lambda$ hyperon in a consistent manner. In fact the model has been proved successful in explaining for the first time an intruder peak observed at considerably low excitation energy in the ${ }^{10} \mathrm{~B}\left(e, e^{\prime} K^{+}\right){ }_{\Lambda}^{10} \mathrm{Be}$ experiment. Here the extended shell model is applied to study novel structures of ${ }_{\Lambda}^{11} \mathrm{~B}$ and ${ }_{\Lambda}^{11} \mathrm{Be}$. In these hypernuclear eigenstates, we also found such new states that the mixing of different-parity nuclear excited states is induced by the participation of a $\Lambda$ hyperon. The wave functions obtained in the extended model space calculations are used to predict the DWIA cross sections for the ${ }^{11} \mathrm{~B}\left(K^{-}, \pi^{-}\right){ }_{\Lambda}^{11} \mathrm{~B}$ and ${ }^{11} \mathrm{~B}\left(\gamma, K^{+}\right){ }_{\Lambda}^{11} \mathrm{Be}$ reactions, and discuss that such unique states should be possibly observed in the high-resolution reaction spectroscopy. We also evaluate the M1, E2 and E1 transtion probabilities among the excited states of the produced hypernuclei. These predictions will be useful in future spectroscopic study of hypernuclear structure when the detailed coincidence measurements of $\left(\pi^{+}, K^{+} \gamma\right)$ and $\left(K^{-}, \pi^{-} \gamma\right)$ reactions are realized in the beamline upgrade planned at the J-PARC facility.

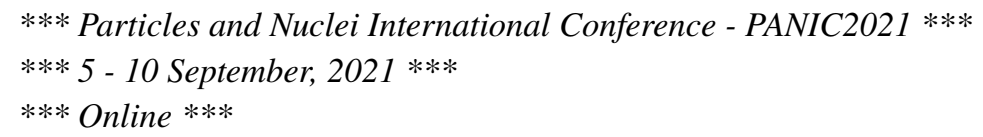

${ }^{*}$ Speaker 


\section{Introduction}

In hypernuclear spectroscopy, different types of production cross sections are often compared in order to elucidate properties of many-body structures. We focus our attention on the understanding of the new results of high-resolution $\left(e, e^{\prime} K^{+}\right)$reaction experiments done at the Jefferson Laboratory (JLab) and then we will also discuss possibility of high-resolution $\left(\pi^{+}, K^{+}\right)$and $\left(K^{-}, \pi^{-}\right)$reactions being planned in the upgrade proposal of the J-PARC beamlines.

Recent $\left(e, e^{\prime} K^{+}\right)$reaction experiments for $p$-shell nuclei [1] done at JLab have confirmed the major peaks predicted by the DWIA calculations [2] based on the normal-parity nuclear-core wave functions coupled with a $\Lambda$ hyperon. At the same time, the data have reported some extra subpeaks which cannot be explained within these conventional wave functions. To describe the extra subpeaks, we have extended the model space by introducing the new configuration which includes non-normal parity nuclear core-excited states $[3,4]$. By this extension we emphasize that the $\Lambda$ hyperon induces intershell mixing of the nuclear core-excited states having different parities.

In this study, for the ${ }_{\Lambda}^{11} \mathrm{~B}$ and ${ }_{\Lambda}^{11} \mathrm{Be}$ hypernuclei, we calculate the energy levels and the DWIA cross sections of $\left(K^{-}, \pi^{-}\right)$and $\left(\gamma, K^{+}\right)$reactions within the framework of an extended model space. Also, we evaluate the M1, E2 and E1 transition strengths for the J-PARC physics projects.

\section{Extended model space for hypernuclei}

Here we employ an extended model space for ${ }_{\Lambda}^{11} \mathrm{~B}\left({ }_{\Lambda}^{11} \mathrm{Be}\right)$ by taking four types of configurations,

$$
\left[J_{\mathrm{c}(i)}^{+} \otimes s^{\Lambda}\right]_{J^{+}},\left[J_{\mathrm{c}(i)}^{+} \otimes p^{\Lambda}\right]_{J^{-}},\left[J_{\mathrm{c}(i)}^{-} \otimes s^{\Lambda}\right]_{J^{-}},\left[J_{\mathrm{c}(i)}^{-} \otimes p^{\Lambda}\right]_{J^{+}},
$$

where $J^{ \pm}$denotes the spin and parity of hypernuclei, and $s^{\Lambda}$ and $p^{\Lambda}$ denote the single-particle states of $\Lambda$ hyperon. $J_{\mathrm{c}(i)}^{+}$denotes the positive-parity states of core-nucleus given by $0 \hbar \omega$ configurations, $(0 s)^{4}(0 p)^{6} . J_{\mathrm{c}(i)}^{-}$denotes the negative-parity states given by $1 p-1 h 1 \hbar \omega$ configurations, $(0 s)^{3}(0 p)^{7}$ $\oplus(0 s)^{4}(0 p)^{5}(s d)^{1}$, in which the spurious center-of-mass excitation is removed in the actual calculations. In this study, the four types configurations are fully taken into account in the model space. It is remarked, however, that the last two types of unnatural-parity core-excited configurations $\left(J_{\mathrm{c}}^{-}\right)$ have been neglected so far in all the conventional shell model calculations. In the negative-parity $\left(J^{-}\right)$states of ${ }_{\Lambda}^{11} \mathrm{~B}\left({ }_{\Lambda}^{11} \mathrm{Be}\right)$, the energy difference between the $s^{\Lambda}$ and $p^{\Lambda}$ states is $1 \hbar \omega$, and that between the $J_{\mathrm{c}}^{+}$and $J_{\mathrm{c}}^{-}$core-states is also $1 \hbar \omega$. Thus, natural- and unnatural-parity nuclear-core configurations can be mixed easily by the $\Lambda N$ interaction at appropriate excitation energy [3, 4].

\section{Results and discussion}

In Fig. 1, for the low-lying levels of ${ }_{\Lambda}^{11} \mathrm{~B}$, the numerical results in the extended shell model are compared with those of the conventional shell model [5] and the experiments. It is noted that the isospin $T=1$ states in Fig. 1 correspond to the states of ${ }_{\Lambda}^{11} \mathrm{Be}$. As for the low-lying states of ${ }_{\Lambda}^{11} \mathrm{~B}$ and the core nucleus ${ }^{10} \mathrm{~B}$, the calculated results (the second and first columns) are in good agreement with the experimental data (the forth and fifth columns), respectively. The average energy of the 2nd doublet $\left(1 / 2^{+}, 3 / 2^{+}\right)$is almost the same as the result of conventional shell model, although the theoretical position is about $300 \mathrm{keV}$ lower than the experimental result. For this doublet, an effect of the spin-orbit term of the $\Lambda N$ interaction is suggested by Millener [5]. 


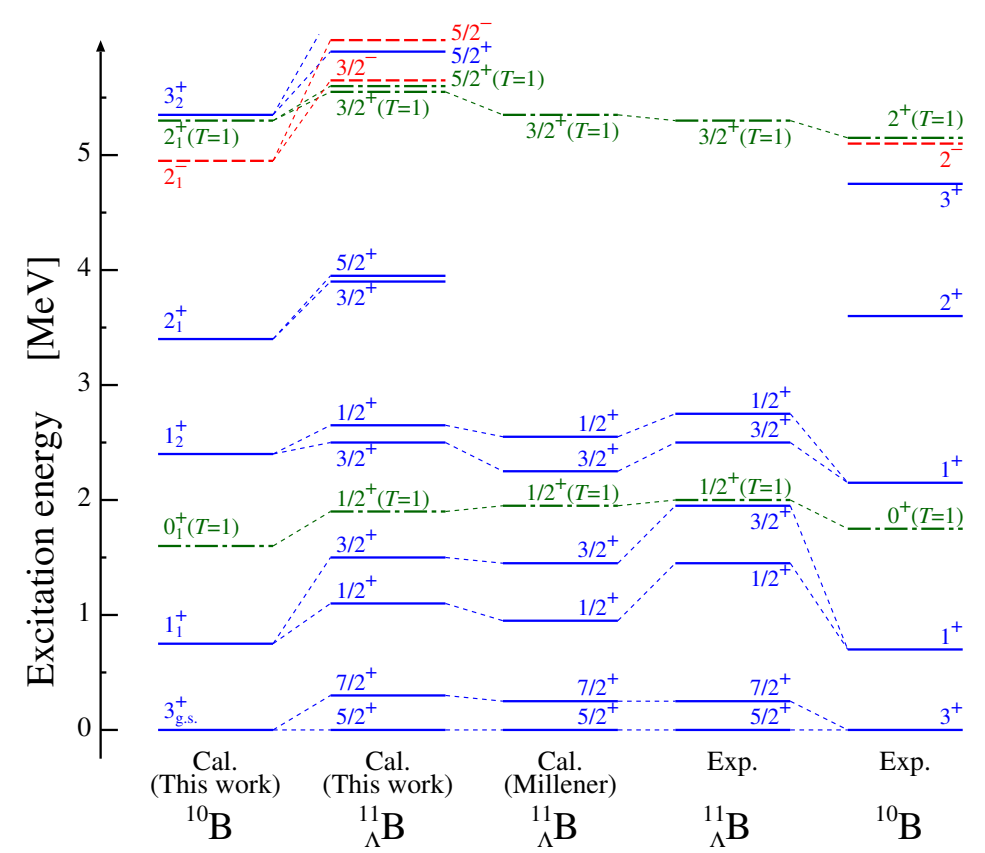

Figure 1: Energy levels for the low-lying states of ${ }_{\Lambda}^{11} \mathrm{~B}$ together with the ${ }^{10} \mathrm{~B}$ core nucleus. The first and second columns show our results, and the third column shows the results of the conventional shell model [5]. The forth and fifth columns show the experimental data.

By using the wave functions of ${ }_{\Lambda}^{11} \mathrm{~B}$ and ${ }_{\Lambda}^{11} \mathrm{Be}$ obtained in the extended model space, we calculate the DWIA cross sections of the $\left(K^{-}, \pi^{-}\right)$and $\left(\gamma, K^{+}\right)$reactions. The panels (a) and (b) of Fig. 2 show the cross sections of the ${ }^{11} \mathrm{~B}\left(K^{-}, \pi^{-}\right)$reactions with incident momenta of $0.80 \mathrm{GeV} / c$ and $1.80 \mathrm{GeV} / c$, respectively. The kinematics of (a) correspond to the recoilless hyperon production in which we can find the substitutional states $\left(3 / 2^{-}\right)$of the surface neutron in the target. The panel (c) shows the cross sections of the ${ }^{11} \mathrm{~B}\left(\gamma, K^{+}\right)$reaction with incident energy of $1.56 \mathrm{GeV}$, which corresponds to the $\left(e, e^{\prime} K^{+}\right)$reaction kinematics adopted recently in the JLab experiments. The solid bars and dashed bars denote the positive- and negative-parity states with $T=0$. The dash-dot bars and dotted bars denote the positive- and negative-parity states with $T=1$. These strength functions do not include contribution from high-lying quasi-free components. For several peaks in Fig. 2, we made component analyses of the main configurations. In the peaks (A), (B) and (C) of panel (b), probabilities of the main configurations ${ }^{10} \mathrm{~B}\left(3_{\text {g.s. }}^{+}\right) \otimes s_{1 / 2}^{\Lambda},{ }^{10} \mathrm{~B}\left(1_{2}^{+}\right) \otimes s_{1 / 2}^{\Lambda}$ and ${ }^{10} \mathrm{~B}\left(2_{1}^{+} ; T=1\right) \otimes s_{1 / 2}^{\Lambda}$ are $99.5 \%, 98.0 \%$ and $99.3 \%$, respectively. These states are almost described by one configuration with $s^{\Lambda}$, respectively, based on the weak coupling. In panel (a), the large peak (D) corresponds to the substitutional state, which mainly consists of three configurations, ${ }^{10} \mathrm{~B}\left(3_{\mathrm{g} . \mathrm{s}}^{+}\right) \otimes p_{3 / 2}^{\Lambda},{ }^{10} \mathrm{~B}\left(1_{2}^{+}\right) \otimes p_{1 / 2}^{\Lambda}$ and ${ }^{10} \mathrm{~B}\left(3_{2}^{+}\right) \otimes p_{3 / 2}^{\Lambda}$ with mixing probabilities of $51.4 \%, 23.0 \%$ and $9.4 \%$. We note that, in the case of $p^{\Lambda}$ states, the $\Lambda N$ interaction causes a kind of strong coupling.

It is quite interesting to see the wave functions for the peaks (E) and (F) of panel (a). They are expressed in terms of the core eigen wave functions coupled with $\Lambda$ as:

$$
\begin{aligned}
& \Psi\left(3 / 2^{-} ; T=0\right)^{(\mathrm{E})}=\sqrt{0.583}\left[{ }^{10} \mathrm{~B}\left(2_{3}^{-}\right) \otimes s_{1 / 2}^{\Lambda}\right]+\sqrt{0.215}\left[{ }^{10} \mathrm{~B}\left(1_{1}^{+}\right) \otimes p_{1 / 2}^{\Lambda}\right]+\cdots, \\
& \Psi\left(3 / 2^{-} ; T=0\right)^{(\mathrm{F})}=\sqrt{0.316}\left[{ }^{10} \mathrm{~B}\left(2_{3}^{-}\right) \otimes s_{1 / 2}^{\Lambda}\right]-\sqrt{0.469}\left[{ }^{10} \mathrm{~B}\left(1_{1}^{+}\right) \otimes p_{1 / 2}^{\Lambda}\right]+\cdots,
\end{aligned}
$$



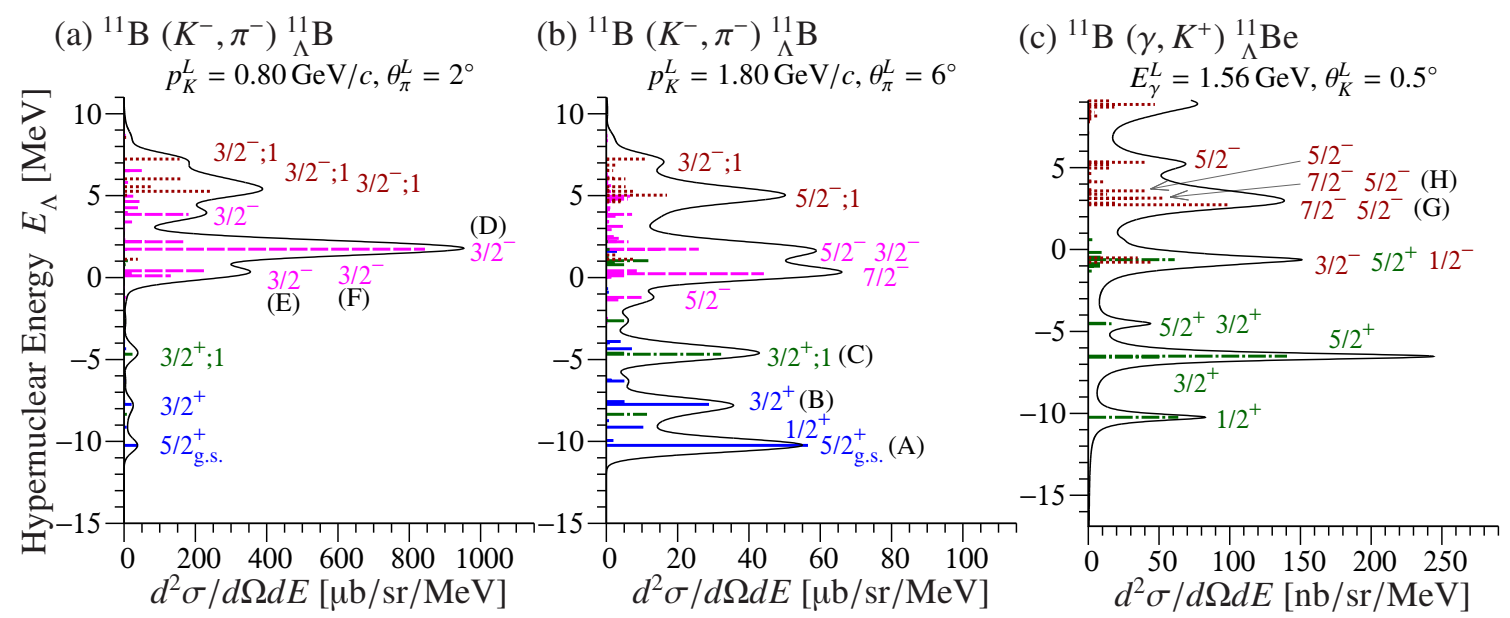

Figure 2: The DWIA cross sections of the $\left(K^{-}, \pi^{-}\right)$reactions with incident momenta of $0.80 \mathrm{GeV} / c$ (a) and $1.80 \mathrm{GeV} / c(\mathrm{~b})$, and $\left(\gamma, K^{+}\right)$reaction with incident energy of $1.56 \mathrm{GeV}$ (c).

and there are large mixings between the natural- and unnatural-parity nuclear-core states. We emphasize that these results are non-trivial ones and are newly obtained in the present extended shell model. The similar mixings are seen in the peaks $(\mathrm{G})$ and $(\mathrm{H})$ of panel $(\mathrm{c})$, which correspond to the ${ }_{\Lambda}^{11} \mathrm{Be}$ states. The states are written by

$$
\begin{aligned}
& \Psi\left(5 / 2^{-} ; T=1\right)^{(\mathrm{G})}=\sqrt{0.737}\left[{ }^{10} \mathrm{Be}\left(3_{3}^{-}\right) \otimes s_{1 / 2}^{\Lambda}\right]-\sqrt{0.179}\left[{ }^{10} \mathrm{Be}\left(2_{1}^{+}\right) \otimes p_{1 / 2}^{\Lambda}\right]+\cdots \\
& \Psi\left(5 / 2^{-} ; T=1\right)^{(\mathrm{H})}=\sqrt{0.212}\left[{ }^{10} \mathrm{Be}\left(3_{3}^{-}\right) \otimes s_{1 / 2}^{\Lambda}\right]+\sqrt{0.420}\left[{ }^{10} \mathrm{Be}\left(2_{1}^{+}\right) \otimes p_{1 / 2}^{\Lambda}\right]+\cdots
\end{aligned}
$$

As in the case of ${ }_{\Lambda}^{11} \mathrm{~B}$, they are newly obtained in the present extended shell model.

For hypernuclei which generally have more bound states than in normal nuclei, even in the lowlying levels many electromagnetic transitions may occur. Table 1 shows the transition strengths for the ${ }_{\Lambda}^{11} \mathrm{~B}$ hypernucleus, $B(\mathrm{M} 1), B(\mathrm{E} 2)$ and $B(\mathrm{E} 1)$, which have large values in each transition. In the M1 transitions, the large $B(\mathrm{M} 1)$ values are seen in the isovector transitions, which occurred between $T=0$ and $T=1$ states. It is noted that the core $\mathrm{M} 1$ transition $B_{\mathrm{c}}\left({ }^{10} \mathrm{~B}, \mathrm{M} 1 ; 0_{1}^{+} ; 1 \rightarrow 1_{1}^{+} ; 0\right)=12.6 \mu_{N}^{2}$ (experimental value of $\left.7.6 \mu_{N}^{2}\right)$ is shared by $B\left(\mathrm{M} 1 ; 1 / 2^{+} ; 1(1.89) \rightarrow 1 / 2^{+} ; 0(1.11)\right)=4.29 \mu_{N}^{2}$ and $B\left(\mathrm{M} 1 ; 1 / 2^{+} ; 1(1.89) \rightarrow 3 / 2^{+} ; 0(1.51)\right)=8.19 \mu_{N}^{2}$ in the extended model calculation. In

Table 1: The $\gamma$ transition strengths $B(\mathrm{M} 1), B(\mathrm{E} 2)$ and $B(\mathrm{E} 1)$ for ${ }_{\Lambda}^{11} \mathrm{~B}$ (in units of $\mu_{N}^{2}, e^{2} \mathrm{fm}^{4}$ and $e^{2} \mathrm{fm}^{2}$ ). The

\begin{tabular}{|c|c|c|c|c|}
\hline$J_{i}^{\pi} ; T_{i}\left(E_{x ; i}\right) \rightarrow J_{f}^{\pi} ; T_{f}\left(E_{x ; f}\right)$ & $B(M 1)$ & $J_{i}^{\pi} ; T_{i}\left(E_{x ; i}\right)$ & $\rightarrow J_{f}^{\pi} ; T_{f}\left(E_{x ; f}\right)$ & $B(E 2) B(E 1)$ \\
\hline $1 / 2^{+} ; 1(1.89) \rightarrow 1 / 2^{+} ; 0(1.11)$ & 4.29 & $1 / 2^{+} ; 0(1.11)$ & $\rightarrow 5 / 2^{+} ; 0(0.00)$ & 6.84 \\
\hline$\rightarrow 3 / 2^{+} ; 0(1.51)$ & 8.19 & $1 / 2^{+} ; 0(2.67)$ & $\rightarrow 3 / 2^{+} ; 0(2.50)$ & 8.83 \\
\hline $5 / 2^{+} ; 0(5.90) \rightarrow 3 / 2^{+} ; 1(5.56)$ & 4.10 & $3 / 2^{+} ; 0(3.93)$ & $\rightarrow 3 / 2^{+} ; 0(1.51)$ & 7.70 \\
\hline $7 / 2^{+} ; 0(6.18) \rightarrow 5 / 2^{+} ; 1(5.63)$ & 4.40 & $5 / 2^{+} ; 0(3.99)$ & $\rightarrow 3 / 2^{+} ; 0(1.51)$ & 9.52 \\
\hline $5 / 2^{+} ; 1(7.61) \rightarrow 7 / 2^{+} ; 0(0.29)$ & 4.52 & $3 / 2^{+} ; 1(5.56)$ & $\rightarrow 1 / 2^{+} ; 1(1.89)$ & 9.54 \\
\hline $3 / 2^{+} ; 1(7.63) \rightarrow 5 / 2^{+} ; 0(0.00)$ & 4.70 & $5 / 2^{+} ; 1(5.63)$ & $\rightarrow 1 / 2^{+} ; 1(1.89)$ & 9.62 \\
\hline $1 / 2^{-} ; 1(8.02) \rightarrow 3 / 2^{-} ; 0(5.66)$ & 6.78 & $3 / 2^{-} ; 0(10.35)$ & $\rightarrow 1 / 2^{+} ; 0(1.11)$ & 0.018 \\
\hline $3 / 2^{-} ; 1(8.02) \rightarrow 5 / 2^{-} ; 0(5.97)$ & 6.59 & $3 / 2^{-} ; 0(10.66)$ & $\rightarrow 1 / 2^{+} ; 0(1.11)$ & 0.016 \\
\hline
\end{tabular}
energies $E_{x}$ are in unit of $\mathrm{MeV}$. The numerical results with large strengths are shown for each transition. 
the weak coupling limit, the ratio for this share is proportional to the squared Racah coefficients, $1: 2$. On the other hand, in the $\mathrm{E} 2$ transitions, the large $B(\mathrm{E} 2)$ values are seen in the isoscalar transitions, which occurred between states with the same isospin. The large value of $B\left(\mathrm{E} 2 ; 1 / 2^{+} ; 0(1.11) \rightarrow 5 / 2^{+} ; 0(0.00)\right)=6.84 e^{2} \mathrm{fm}^{4}$ mainly consists of the core E2 transition $B_{\mathrm{c}}\left({ }^{10} \mathrm{~B}, \mathrm{E} 2 ; 1_{1}^{+} ; 0 \rightarrow 3_{\mathrm{g} . \mathrm{s} .}^{+} ; 0\right)=7.1 e^{2} \mathrm{fm}^{4}$ (experimental value of $\left.4.2 e^{2} \mathrm{fm}^{4}\right)$. In the purely simplified picture, these $B(\mathrm{E} 2)$ strengths have the same value, $B(\mathrm{E} 2 ; 1 / 2 \rightarrow 5 / 2)=B_{\mathrm{c}}\left({ }^{10} \mathrm{~B}, \mathrm{E} 2 ; 1 \rightarrow 3\right)$. The extended model calculation shows the slight shrinkage of core nucleus by the glue like role of $\Lambda$. It is noted that $B\left(\mathrm{E} 2 ; 1 / 2^{+} ; 0(2.67) \rightarrow 3 / 2^{+} ; 0(2.50)\right)$ has the large value of $8.83 e^{2} \mathrm{fm}^{4}$. In this transition, the initial and final states are doublet partners based on the common nuclear-core state ${ }^{10} \mathrm{~B}\left(1_{2}^{+}\right)$, and the $B(\mathrm{E} 2)$ value in these states consists of the electric quadrupole moment of the nuclear-core state. We point out a possibility that the quadrupole moments of excited states of core nuclei can be estimated by such $B(\mathrm{E} 2)$ values to be measured through hypernuclear experiments.

We remark the $\mathrm{E} 1$ transitions from parity-mixing states in ${ }_{\Lambda}^{11} \mathrm{~B}$. In the E1 transitions in Table 1 (at right-bottom $)$, the initial states $3 / 2^{-} ; T=0\left(E_{x}=10.35 \mathrm{MeV}\right)$ and $3 / 2^{-} ; T=0\left(E_{x}=10.66 \mathrm{MeV}\right)$ are parity-mixed states (cf. Eqs. (1) and (2)), which correspond to the peaks (E) and (F) in Fig. 2 (a). The $B(\mathrm{E} 1)$ values of $0.018 e^{2} \mathrm{fm}^{2}$ and $0.016 e^{2} \mathrm{fm}^{2}$ are obtained by using the conventional $\Lambda$ effective charge, $e_{\Lambda}^{\mathrm{eff}}=-Z e / A=-5 e / 11$. In these states, the configuration with $J_{\mathrm{c}}^{\pi}=1_{2}^{+}$core and $p_{1 / 2}^{\Lambda}$ mainly contributes to the $B(\mathrm{E} 1)$ strengths. The $p_{1 / 2}^{\Lambda}$ single-particle state goes down to the $s^{\Lambda}$ state, and the nuclear-core state does not change in the E1 transition, ${ }^{10} \mathrm{~B}\left(1_{2}^{+}\right) \otimes p_{1 / 2}^{\Lambda} \rightarrow{ }^{10} \mathrm{~B}\left(1_{2}^{+}\right) \otimes s_{1 / 2}^{\Lambda}$. We also emphasize that the configuration with large E1 strength splits into the two states due to the nuclear-core parity-mixing by $\Lambda N$ interaction. The structures of hypernuclei and/or core nuclei will be elucidated by the measurements of M1, E2 and E1 transitions in high-resolution $\left(\pi^{+}, K^{+} \gamma\right)$ and $\left(K^{-}, \pi^{-} \gamma\right)$ reactions being planned in the upgrade proposal of the J-PARC beamlines.

\section{Concluding remarks and acknowledgements}

We have calculated the energy levels and the M1, E2 and E1 transition rates for hypernuclei ${ }_{\Lambda}^{11} \mathrm{~B}\left({ }_{\Lambda}^{11} \mathrm{Be}\right)$ by the extended shell model. The obtained wave functions are used to estimate the DWIA cross sections of the $\left(K^{-}, \pi^{-}\right)$and $\left(\gamma, K^{+}\right)$reactions, which show large cross sections for the particular states that embody the intershell mixing of the nuclear core-excited states having different parities. The parity-mixing can affect E1 transition strengths as well. The major conclusions are summarized in the Abstract.

This work was supported by JSPS KAKENHI Grant Number JP20K03950.

\section{References}

[1] T. Gogami et al., Phys. Rev. C 93 (2016) 034314, and references therein.

[2] T. Motoba et al., Prog. Theor. Phys. Suppl. 117 (1994) 123.

[3] A. Umeya, T. Motoba, K. Itonaga, JPS Conf. Proc. 26 (2019) 023016.

[4] A. Umeya, T. Motoba, K. Itonaga, J. Phys. Conf. Ser. 1643 (2020) 012110.

[5] D. J. Millener, Nucl. Phys. A 804 (2008) 84. 